\title{
Explanation of the Hidden Variables in the Electron-Positron Annihilation Process in Terms of the Quantum Entanglement and the Conservation of Flux Quantum
}

\author{
Mesude Saglam¹, Hanaslı Gür², Oktay Yilmaz \\ ${ }^{1}$ Department of Physics, Ankara University, Ankara, Turkey \\ ${ }^{2}$ Department of Engineering Physics, Ankara University, Ankara, Turkey \\ ${ }^{3}$ Department of Physics, Faculty of Arts and Sciences, Çanakkale Onsekiz Mart University, Terzioglu Campus, \\ Canakkale, Turkey \\ Email: saglam@science.ankara.edu.tr
}

How to cite this paper: Saglam, M., Gür, H. and Yilmaz, O. (2018) Explanation of the Hidden Variables in the Electron-Positron Annihilation Process in Terms of the Quantum Entanglement and the Conservation of Flux Quantum. Journal of Modern Physics, 9, 985-996.

https://doi.org/10.4236/jmp.2018.95061

Received: March 9, 2018

Accepted: April 14, 2018

Published: April 17, 2018

Copyright $\odot 2018$ by authors and Scientific Research Publishing Inc. This work is licensed under the Creative Commons Attribution International License (CC BY 4.0).

http://creativecommons.org/licenses/by/4.0/

\begin{abstract}
We show that the electron-positron annihilation process resulting with the creation of two gamma photons cannot be fully determined without the conservation of the angular momentum which has two elements, namely, the conservation of the spin angular momentum and the conservation of the quantum flux which work as the conservation of the magnetic moments as well. The conservation of the quantum flux has never been considered so far for any collision process. We show that the missing conservation rule in the above process is the conservation of the total quantum flux which is the hidden variable of that process. By using the quantum entanglement together with the conservation of the quantum flux we show that the initial and the final states of this collision are fully determined. We also show that each of the gamma photons created in the end carries a quantum flux of $\pm \Phi_{0}= \pm h c / e$ with itself along the propagation direction. Here the $(+)$ and (-) signs correspond to the right hand and left circular helicity, respectively.
\end{abstract}

\section{Keywords}

Hidden Variable, Dirac Equation, Spin, Quantum Flux, Electron-Positron Annihilation, Right (Left) Hand Circular Helicity

\section{Introduction}

Electron-positron annihilation process has been known since the year 1930. 
After the collision we have two gamma photons with the same energy $E=m_{0} c^{2}=\hbar \omega$ but with different helicities. Here $m_{0}$ is the rest mass of the electron $\left(e^{-}\right)$and the positron $\left(e^{+}\right)$and $\omega$ is the angular frequencies of the created photons. The aim of the present work is to show that the electron-positron annihilation process resulting with the creation of two gamma photons cannot be fully determined without the conservation of the angular momentum which has two elements, namely, the conservation of the spin angular momentum and the conservation of the quantum flux. The conservation of the quantum flux has never been considered so far for any collision process. We show that the missing conservation rule in the above process is the conservation of the total quantum flux which corresponds to the hidden variable in that process. By using the quantum entanglement together with the conservation of the quantum flux we show that the initial and the final states of this collision are fully determined. We also show that each of the gamma photons created in the end carries a quantum flux of $\pm \Phi_{0}= \pm h c / e$ with itself along the propagation direction. Here the (+) and $(-)$ signs correspond to the right hand and left circular helicity, respectively. Since the quantum flux is essential for the hidden variables, we give a summary of related works so far. Magnetic flux quantization has been known since London [1] and Onsager [2]. Recently Saglam and Boyacioglu [3] calculated semiclassically, the quantized magnetic flux through the Landau orbits of an electron including the effect of the spinning motion. They showed that the spin contribution to the quantized flux is equal to $\left( \pm \frac{1}{2} \Phi_{0}\right)$ depending on the spin orientation. Here $\Phi_{0}=h c / e=4.14 \times 10^{-7} \mathrm{G} \cdot \mathrm{cm}^{2}$. Wan and Saglam [4] calculated quantum mechanically the intrinsic magnetic flux of an electron due to its orbital motion in a non-relativistic hydrogen atom by using the Schödinger equation and then extended their result to incorporate the spin angular momentum as well. They showed that the intrinsic magnetic flux quanta coming from the quantum numbers $m_{l}$ and $m_{s}$ are $\left(-m_{l} \Phi_{0}\right)$ and $\left(-m_{s} \Phi_{0}\right)$ respectively. It was first time pointed out by Wan [5] that the intrinsic quantum flux of electron must be related to the hidden variables in quantum mechanics. In Appendix I we show that the conservation of the angular momentum has two elements, namely, the conservation of the spin angular momentum and the conservation of the quantum flux. Since later one has not been considered so far in any collision, we argue that the conservation of the quantum flux in any collision must be related to the hidden variables in quantum mechanics. In Appendix II we show that the conservation of the quantum flux can be replaced by the conservation of the magnetic moments as well. As the subject of present study is related to the intrinsic fluxes of $\left(e^{-}\right)$and $\left(e^{+}\right)$we need to calculate these fluxes in the relativistic sense. Although Wan and Saglam [4] estimated the intrinsic magnetic flux of an electron to be $\left( \pm \frac{1}{2} \Phi_{0}\right)$ by using the non-relativistic Schödinger equation, a rigorous proof has been needed also. In Appendix III we calculate the intrinsic fluxes of $\left(e^{-}\right)$and $\left(e^{+}\right)$by the spin dependent solutions [6] of the Dirac equation 
for free electron (or positron) in a uniform magnetic field. We then prove that the intrinsic fluxes of $\left(e^{-}\right)$and $\left(e^{+}\right)$are $\left( \pm \frac{1}{2} \Phi_{0}\right)$ depending on the spin orientation. The outline of this paper is as follows: In Section 2 we explain the need for hidden variables in quantum mechanics. In Section 3 we find the exact initial and final state wave functions of the electron-positron annihilation process by using the conservation of the spin angular momentum and the conservation of quantum flux which can be replaced by the conservation of the magnetic moments. In Section 4 we give the conclusions.

\section{The Need for Hidden Variables in the Electron-Positron Annihilation Process}

The electron-positron annihilation occurs when an electron $\left(e^{-}\right)$and a positron $\left(e^{+}\right)$collide.

After the collision we have two gamma photons with the same energy, $E=m_{0} c^{2}=\hbar \omega$. Here $m_{0}$ is the rest mass of both $\left(e^{-}\right.$and $\left.e^{+}\right)$and $\omega$ is the angular frequencies of the gamma photons. In this collision we have the conservation of charge, energy, linear momentum and the angular momentum. It has been known that the above conservation rules are not adequate to determine the final state quantum mechanically. Therefore an extra conservation rule which is the hidden variables in this case, is needed. We will show that the missing conservation rule has been the conservation of the total quantum flux which is essentially a component of the angular momentum conservation law (Appendix I).

To proceed further, we will assume that electron and positron collide at the origin of the coordinate system. After the collision we will have two photons with the same energy:

$$
E=m_{0} c^{2}=\hbar \omega=\hbar k c=h c / \lambda
$$

Here $m_{0} c^{2}$ is the rest mass energy of both $e^{-}$and $e^{+}$and $\hbar \omega$ is energy of the each gamma photon with the angular frequency $\omega$. Just before the collision the relative spin orientation of $e^{-}$and $e^{+}$will be controlled by the Heisenberg exchange Hamiltonian [7] [8]. If we had two electrons instead, the exchange energy would have the form:

$$
U_{\text {exc }}\left(e^{-}, e^{+}\right)=-2 J\left(e^{-}, e^{+}\right) \boldsymbol{S}_{1} \cdot \boldsymbol{S}_{2}
$$

where $S_{1}$ and $S_{2}$ are the spin vectors of these two electrons and $J\left(e^{-}, e^{-}\right)$ is the exchange integral which is a positive quantity for the repulsive Coulomb potentials [8] resulting that Heisenberg Exchange Hamiltonian $U_{\text {exc }}$ has a minumum eigenvalue for opposite spins. But for an electron-positron $\left(e^{-}, e^{+}\right)$ pair the exchange Hamiltonian has the opposite sign:

$$
U_{\text {exc }}\left(e^{-}, e^{+}\right)=2 J\left(e^{-}, e^{+}\right) \boldsymbol{S}_{1} \cdot \boldsymbol{S}_{2}
$$

where $S_{1}$ and $S_{2}$ are the spin vectors of electron's and positron's respectively. This time because of the attractive Coulomb potential between $e^{-}$and $e^{+}$the exchange integral $J=J\left(e^{-}, e^{+}\right)$will be a negative quantity: $J=-|J|$ resulting 
$U_{e x c}=-2 J S_{1} \cdot S_{2}$ which has a minimum energy eigenvalue for parallel spins. Therefore just before the collision the total $z$-component of the spin of the colliding $\left(e^{-}, e^{+}\right)$system will be \pm 1 . That means there are two possibilities:

1) electron is in spin-down state and positron is in spin-down state.

2) electron is in spin-up state and positron is in spin-up state.

In Dirac notation the eigenstates (a) and (b) can be defined as:

$$
\begin{gathered}
\phi_{a}=\left|e^{-} \downarrow\right\rangle\left|e^{+} \downarrow\right\rangle \\
\phi_{b}=\left|e^{-\uparrow}\right\rangle\left|e^{+} \uparrow\right\rangle .
\end{gathered}
$$

The $\mathrm{z}$-components of the spin for the eigenstates (a) and (b) given in Equations (4a) (4b) are:

$$
\begin{aligned}
& \left(\sum S_{z}\right)_{a}=-\frac{1}{2}-\frac{1}{2}=-1 \\
& \left(\sum S_{z}\right)_{b}=\frac{1}{2}+\frac{1}{2}=1 .
\end{aligned}
$$

Since the states (a) and (b) are equally probable, the total initial wave function $\Psi_{i}$ before the collision can be written as in two forms; Namely:

$$
\begin{gathered}
\Psi_{\text {initial- } 1}=\frac{1}{\sqrt{2}}\left(\phi_{a}+\phi_{b}\right)=\frac{1}{\sqrt{2}}\left(\left|e^{-} \downarrow\right\rangle\left|e^{+} \downarrow\right\rangle+\left|e^{-\uparrow}\right\rangle\left|e^{+} \uparrow\right\rangle\right) \\
\Psi_{\text {initial-2 }}=\frac{1}{\sqrt{2}}\left(\phi_{a}-\phi_{b}\right)=\frac{1}{\sqrt{2}}\left(\left|e^{-} \downarrow\right\rangle\left|e^{+} \downarrow\right\rangle-\left|e^{-\uparrow}\right\rangle\left|e^{+} \uparrow\right\rangle\right)
\end{gathered}
$$

which are the quantum entanglement of the states $\left|e^{-} \downarrow\right\rangle,\left|e^{+} \downarrow\right\rangle,\left|e^{-\uparrow}\right\rangle$ and $\left|e^{+} \uparrow\right\rangle$. The expectation value of $\sum S_{z}$ (the total z-component of the spin) for the initial states in Equations (6a) and (6b) are:

$$
\begin{aligned}
& \left\langle\sum S_{z}\right\rangle_{\text {initial-1 }}=\frac{1}{2}\left(\sum S_{z}\right)_{a}+\left(\sum S_{z}\right)_{b}=\frac{1}{2}(-1+1)=0 \\
& \left\langle\sum S_{z}\right\rangle_{\text {initial-2 }}=\frac{1}{2}\left[\left(\sum S_{z}\right)_{a}-\left(\sum S_{z}\right)_{b}\right]=\frac{1}{2}(-1-1)=-1
\end{aligned}
$$

The total wave function, $\Psi_{f}$ of the system after collision, will correspond to another entanglement of the states $\left|e^{-} \downarrow\right\rangle,\left|e^{+} \downarrow\right\rangle,\left|e^{-\uparrow}\right\rangle$ and $\left|e^{+\uparrow}\right\rangle$ provided that all the conservation rules are met. Again there are two possibilities for the final wave function. These are:

$$
\begin{aligned}
& \Psi_{\text {final- }}=\frac{1}{\sqrt{2}}\left(\phi_{a}^{\prime}+\phi_{b}^{\prime}\right)=\frac{1}{\sqrt{2}}\left(\left|e^{-} \downarrow\right\rangle\left|e^{+} \uparrow\right\rangle+\left|e^{-\uparrow}\right\rangle\left|e^{+} \downarrow\right\rangle\right) \\
& \Psi_{\text {final-2 }}=\frac{1}{\sqrt{2}}\left(\phi_{a}^{\prime}-\phi_{b}^{\prime}\right)=\frac{1}{\sqrt{2}}\left(\left|e^{-} \downarrow\right\rangle\left|e^{+} \uparrow\right\rangle-\left|e^{-\uparrow}\right\rangle\left|e^{+} \downarrow\right\rangle\right)
\end{aligned}
$$

where

$$
\phi_{a}^{\prime}=\left|e^{-} \downarrow\right\rangle\left|e^{+} \uparrow\right\rangle ; \phi_{b}^{\prime}=\left|e^{-\uparrow}\right\rangle\left|e^{+} \downarrow\right\rangle .
$$

The z-components of the spin for the eigenstates $\phi_{a}^{\prime}$ and $\phi_{b}^{\prime}$ of Equation (8c) are: $\left(\sum S_{z}\right)_{a}^{\prime}=0 \quad\left(\sum S_{z}\right)_{b}^{\prime}=0$ respectively. Therefore the expectation 
value of $\sum S_{z}$ for the final states given in Equations (8a) and (8b) are:

$$
\begin{gathered}
\left\langle\sum S_{z}\right\rangle_{\text {final- } 1}=\frac{1}{2}(0+0)=0 \\
\left\langle\sum S_{z}\right\rangle_{\text {final-2 }}=\frac{1}{2}(0-0)=0 .
\end{gathered}
$$

If we compare the total z-component of the spin before and after the collision, Equations (7a) (7b) and Equations (9a) (9b), we see that the conservation of the spin angular momentum excludes the initial wave function $\left(\Psi_{\text {initial-2 }}\right)$ given in Equation (6b) as it has total z-component of spin equal to $(-1)$ but not zero. However the conservation of the spin angular momentum does not distinguish the final states given in Equation (8a) and (8b) as they have the same $z$-component of spin given in Equations (9a) (9b). Therefore there must be another conservation rule (besides the conservation of charge, energy, linear and spin angular momentum) that fully determines the final state. In Appendix I we will show that the conservation of the angular momentum requires also the conservation of the flux quantum as well. Namely:

Total flux quanta before the collision $=$ Total flux quanta after the collision

Therefore in all the collisions that the conservation of the spin and the orbital angular momentums are involved, the conservation of the quantum flux is a necessary condition to find the exact wave functions before and after the collision. Therefore the statement given above must contain the hidden variables to determine the final state exactly. In the following section we will show that the conservation of the quantum flux excludes the final wave function $\left(\Psi_{\text {final-2 }}\right)$ given in Equation (8b). So the collision of the electron and the positron resulting with the creation of two gamma photons simply corresponds to the transition from the initial entangled state $\left(\Psi_{\text {initial-1 }}\right)$ to the final entangled state $\left(\Psi_{\text {final-1 }}\right)$; Namely:

$$
\frac{1}{\sqrt{2}}\left(\left|e^{-} \downarrow\right\rangle\left|e^{+} \downarrow\right\rangle+\left|e^{-\uparrow}\right\rangle\left|e^{+} \uparrow\right\rangle\right) \rightarrow \frac{1}{\sqrt{2}}\left(\left|e^{-} \downarrow\right\rangle\left|e^{+} \uparrow\right\rangle+\left|e^{-\uparrow}\right\rangle\left|e^{+} \downarrow\right\rangle\right)
$$

\section{Finding the Exact Initial and Final State Wave Functions of the Electron-Positron Annihilation Process by Using Spin Angular Momentum and the Quantum Flux Conservation}

As was stated in the introduction section and calculated rigorously in Appendix II the intrinsic quantum fluxes that a free electron (or a positron) carries with itself are:

$$
\begin{aligned}
& \Phi\left(e^{-} \downarrow\right)=\frac{h c}{2 e}=\frac{\Phi_{0}}{2} \\
& \Phi\left(e^{-\uparrow}\right)=-\frac{h c}{2 e}=-\frac{\Phi_{0}}{2} \\
& \Phi\left(e^{+} \downarrow\right)=-\frac{h c}{2 e}=-\frac{\Phi_{0}}{2}
\end{aligned}
$$




$$
\Phi\left(e^{+\uparrow}\right)=\frac{h c}{2 e}=\frac{\Phi_{0}}{2} .
$$

Next by using Equations (11)-(14) we can calculate the quantum fluxes of the eigenstates $\phi_{a}$ and $\phi_{b}$ given in Equations (4a) (4 b):

$$
\begin{gathered}
\Phi\left(e^{-}, e^{+}\right)_{a}=\Phi\left(e^{-} \downarrow\right)+\Phi\left(e^{+} \downarrow\right)=\frac{h c}{2 e}-\frac{h c}{2 e}=0 \\
\Phi\left(e^{-}, e^{+}\right)_{b}=\Phi\left(e^{-\uparrow}\right)+\Phi\left(e^{+} \uparrow\right)=-\frac{h c}{2 e}+\frac{h c}{2 e}=0
\end{gathered}
$$

By using Equations (15a) and (15b), the expectation value of the total quantum fluxes for the suggested initial states of Equations (6a) and (6b) are:

$$
\begin{aligned}
& \left\langle\sum \Phi\right\rangle_{\text {initial- } 1}=\frac{1}{2}\left[\Phi\left(e^{-}, e^{+}\right)_{a}+\Phi\left(e^{-}, e^{+}\right)_{b}\right]=\frac{1}{2}(0+0)=0 \\
& \left\langle\sum \Phi\right\rangle_{\text {initial-2 }}=\frac{1}{2}\left[\Phi\left(e^{-}, e^{+}\right)_{a}-\Phi\left(e^{-}, e^{+}\right)_{b}\right]=\frac{1}{2}(0-0)=0 .
\end{aligned}
$$

Next we calculate quantum fluxes of the eigenstates $\phi_{a}^{\prime}$ and $\phi_{b}^{\prime}$ after the collision. By using Equations (11)-(14) and Equation (8c), the related fluxes are:

$$
\begin{gathered}
\Phi\left(e^{-}, e^{+}\right)_{a}^{\prime}=\Phi\left(e^{-} \downarrow\right)+\Phi\left(e^{+} \uparrow\right)=\frac{h c}{2 e}+\frac{h c}{2 e}=\frac{h c}{e}=\Phi_{0} \\
\Phi\left(e^{-}, e^{+}\right)_{b}^{\prime}=\Phi\left(e^{-\uparrow}\right)+\Phi\left(e^{+} \downarrow\right)=-\frac{h c}{2 e}-\frac{h c}{2 e}=-\frac{h c}{e}=-\Phi_{0}
\end{gathered}
$$

Similarly from Equations (17a) (17b), the expectation value of the total quantum fluxes for the suggested final states of Equations (8a) and (8b) are:

$$
\begin{aligned}
& \left\langle\sum \Phi\right\rangle_{\text {final- } 1}=\frac{1}{2}\left[\Phi\left(e^{-}, e^{+}\right)_{a}^{\prime}+\Phi\left(e^{-}, e^{+}\right)_{b}^{\prime}\right]=\frac{1}{2}\left(\Phi_{0}-\Phi_{0}\right)=0 \\
& \left\langle\sum \Phi\right\rangle_{\text {final-2 }}=\frac{1}{2}\left[\Phi\left(e^{-}, e^{+}\right)_{a}^{\prime}-\Phi\left(e^{-}, e^{+}\right)_{b}^{\prime}\right]=\frac{1}{2}\left(\Phi_{0}+\Phi_{0}\right)=\Phi_{0} .
\end{aligned}
$$

As was done in section II, If we compare the total quantum fluxes before and after the collision we see that this time, conservation of the flux quanta excludes the suggested final wave function ( $\Psi_{\text {final-2 }}$ ) given in Equation (8b) as the total quantum flux is equal to $\left(\Phi_{0}\right)$ but not zero as it should be.

In summary, conservation of the spin angular momentum excludes the suggested initial wave function, ( $\Psi_{\text {initial-2 }}$ ) given in Equation (6b) while the conservation of the quantum flux excludes the suggested final wave function ( $\Psi_{\text {final-2 }}$ ) given in Equation (8b). Therefore as was stated in Equation (10), the collision of the electron and the positron resulting with the creation of two gamma photons simply corresponds to the transition from the initial entangled state ( $\left.\Psi_{\text {initial- } 1}\right)$ to the final entangled state ( $\Psi_{\text {final-1 }}$ ).

\section{Conclusions}

We have shown that the electron-positron annihilation process resulting with the creation of two gamma photons cannot be fully determined without the conservation of the angular momentum which has two elements, namely, the 
conservation of the spin angular momentum and the conservation of the quantum flux. The conservation of the quantum flux has never been considered so far for any collision process. We show that the missing conservation rule in the above process is the conservation of the total quantum flux which is the hidden variable of that process. By using the quantum entanglement together with the conservation of the quantum flux we show that the initial and the final states of this collision are fully determined.

The additional results of the present work can be summarized as follows:

1) From Equations (17a) (17b), each of the gamma photons carries a quantum flux of $\pm \Phi_{0}= \pm h c / e$ with itself along the propagation direction. Here the $(+)$ and (-) signs correspond to the right hand and left circular helicity, respectively.

2) The spin of each gamma photon is equal to zero but not unity.

3) The magnetic moment of each gamma photon is $\pm 2 \mu_{B}$ depending on the helicity.

4) Since the above results 1)-3) do not depend on the angular frequency, $\omega$ of the gamma photons, we then expect them to be valid for any photon as well.

A more complete work will be presented in the future.

\section{References}

[1] London, F. (1950) Superfluids. John Wiley and Sons, Hoboken.

[2] Onsager, L. (1954) Diamagnetism in Metals. Proceeding of the International Conference on Theoretical Physics, Kyoto and Tokyo, September 1953, 669-675. Introductory Talk [on Liquid Helium], 877-880. Science Council of Japan, Tokyo.

[3] Saglam, M. and Boyacioglu, B. (2002) International Journal of Modern Physics B, 16, 607. https://doi.org/10.1142/S0217979202010038

[4] Wan, K.K. and Saglam, M. (2006) International Journal of Theoretical Physics, 45, 1132-1151. https://doi.org/10.1007/s10773-006-9118-Z

[5] Wan, K.K. (2006) From Micro to Macro Quantum Quantum Systems: A Unified Tratment with Superselection Rules and Its Applications. Imperial College Press, London. https://doi.org/10.1142/p427

[6] Yilmaz, O., Saglam, M. and Aydin, Z.Z. (2007) The Journal of the Old and New Concepts in Physics, 4, 141. https://doi.org/10.2478/v10005-007-0007-x

[7] Levine, I.N. (2000) Quantum Chemistry. Prentice Hall, Upper Saddle River.

[8] Slater, J.C. (1930) Physical Review, 36, 51. https://doi.org/10.1103/PhysRev.36.57

[9] Lerda, A. (1992) Anyons. Springer-Verlag Heidelberg. 


\section{Appendix I. Conservation of the Quantum Flux}

The Lagrangian of an electron with mass $m_{0}$ and electric charge $(-e)$ moving in a uniform magnetic field in $z$ direction $\boldsymbol{B}=B \hat{z}$ is given by, $L=\frac{1}{2} m_{0} \boldsymbol{v}^{2}-\frac{e}{c} \boldsymbol{v} \cdot \boldsymbol{A}(r)$ where $\boldsymbol{r}=\boldsymbol{r}(x, y)$ is the position vector in two dimensions, $\boldsymbol{v}$ is the velocity and $\boldsymbol{A}$ is the vector potential. The quantum flux in terms of radius and the magnetic field can be written as

$$
\Phi=\oint \frac{\boldsymbol{B}}{2} \cdot(\boldsymbol{r} \times \mathrm{d} \boldsymbol{r})=\oint \frac{\boldsymbol{B}}{2} \cdot\left(\boldsymbol{r} \times \frac{\mathrm{d} \boldsymbol{r}}{\mathrm{d} t}\right) \mathrm{d} t
$$

For an electron moving in the $\mathrm{x}-\mathrm{y}$ plane in the counter clockwise direction with the angular frequency $\omega_{c}=\frac{e B}{m_{0} c}$, we get $\Phi=\pi\left(x^{2}+y^{2}\right) B$, where the time integral has been taken over one cyclic period $T_{c}=\frac{2 \pi}{\omega_{c}}$. The vector potential, $\boldsymbol{A}$ is now related to the magnetic flux $\Phi$ given in Equation (AI-1) by

$$
A(r)=\frac{\Phi}{2 \pi}\left(\frac{-y}{x^{2}+y^{2}} \hat{x}+\frac{x}{x^{2}+y^{2}} \hat{y}\right)
$$

Let us consider the z-component of the conserved canonical angular momentum $J_{c}$ is given [9] by

$$
J_{c}=(\boldsymbol{r} \times \boldsymbol{p})_{z}=\left[\boldsymbol{r} \times\left(m_{0} \boldsymbol{v}-\frac{e}{c} \boldsymbol{A}\right)\right]_{z}=J-e \Phi / 2 \pi c
$$

where $J$ is the gauge invariant kinetic angular momentum. The difference between $J_{c}$ and $J$ is due to presence of the magnetic flux and hence the magnetic field $B$. Both in the absence and the presence of the magnetic field $B$, the canonical angular momentum is always represented by $J_{c}=-i \hbar \partial / \partial \varphi$ and its eigenvalues are $m \hbar(m \in Z)$. So the spectrum of kinetic angular momentum operator $J$ consists of integers shifted by $(e \Phi / h c)$. Therefore Equation (AI-3) simply states that the conservation of the angular momentum requires also the conservation of the flux quantum as well. Namely:

Total flux quanta before the collision $=$ Total flux quanta after the collision

Therefore in all the collisions that the conservation of the spin and the orbital angular momentums are involved, the conservation of the quantum flux is a necessary condition to find the exact wave functions before and after the collision. In the process of electron-positron collision resulting with the creation of two gamma photons, the total flux quanta before the collision must be equal to the total flux quanta after the collision. In Appendix II we will show that the conservation of the quantum flux can be replaced by the conservation of the magnetic moments as well. 


\section{Appendix II: Magnetic Moments of the System before and after the Collision}

We first give the definition of the spin magnetic moment vector, $\boldsymbol{\mu}$ for a free electron $\left(e^{-}\right)$and a positron $\left(e^{+}\right)$respectively:

$$
\begin{gathered}
\boldsymbol{\mu}_{e^{-}}=-g \mu_{B} \boldsymbol{S} \\
\boldsymbol{\mu}_{e^{+}}=g \mu_{B} S
\end{gathered}
$$

Here $\hbar \boldsymbol{S}$ is the spin angular momentum of both electron and positron, $g$ is the Lande factor which is equal to 2 for a free electron (or positron) and $\mu_{B}$ is the Bohr magneton defined as:

$$
\mu_{B}=\frac{e \hbar}{2 m_{0} c} .
$$

Now we calculate the expectation value of $\sum \mu_{z}$ (the total z-component of the magnetic moment) for the initial states given in Equations (6a) and (6b) are:

$$
\begin{aligned}
& \left\langle\sum \mu_{z}\right\rangle_{\text {initial- }-1}=\frac{1}{2}\left[\left(\sum \mu_{z}\right)_{a}+\left(\sum \mu_{z}\right)_{b}\right]=0 \\
& \left\langle\sum \mu_{z}\right\rangle_{\text {initial-2 }}=\frac{1}{2}\left[\left(\sum \mu_{z}\right)_{a}-\left(\sum \mu_{z}\right)_{b}\right]=0
\end{aligned}
$$

The expectation value of $\sum \mu_{z}$ for the final states given in Equations (8a) and $(8 \mathrm{~b})$ are:

$$
\begin{aligned}
& \left\langle\sum \mu_{z}\right\rangle_{\text {final- } 1}=\frac{1}{2}\left(2 \mu_{B}-2 \mu_{B}\right)=0 \\
& \left\langle\sum \mu_{z}\right\rangle_{\text {final- } 2}=\frac{1}{2}\left(2 \mu_{B}+2 \mu_{B}\right)=2 \mu_{B}
\end{aligned}
$$

As was done in section III, if we compare the expectation value of $\sum \mu_{z}$ for the initial states Equations (6a) (6b) and the final states Equations (8a) (8b), we see that conservation of the total magnetic moment works just as the conservation of the flux quantum. Namely both of them excludes the suggested final wave function ( $\Psi_{\text {final-2 }}$ ) given in Equation (8b). Therefore the collision of the electron and the positron resulting with the creation of two gamma photons simply corresponds to the transition from the initial entangled state $\left(\Psi_{\text {initial }-1}\right)$ to the final entangled state ( $\Psi_{\text {final-1 }}$ ); Namely:

$$
\frac{1}{\sqrt{2}}\left(\left|e^{-} \downarrow\right\rangle\left|e^{+} \downarrow\right\rangle+\left|e^{-} \uparrow\right\rangle\left|e^{+} \uparrow\right\rangle\right) \rightarrow \frac{1}{\sqrt{2}}\left(\left|e^{-} \downarrow\right\rangle\left|e^{+} \uparrow\right\rangle+\left|e^{-} \uparrow\right\rangle\left|e^{+} \downarrow\right\rangle\right)
$$

From the final state $\left(\Psi_{\text {final-1 }}\right)$ given in Equation (10) z-component of the magnetic moment of each eigenstates (the gamma photons with the right hand $(r h)$ and the left hand ( $l h)$ circular helicity) are:

$$
\begin{gathered}
\mu_{z}(r h)=2 \mu_{B}=\frac{e \hbar}{m_{0} c} . \\
\mu_{z}(l h)=-2 \mu_{B}=-\frac{e \hbar}{m_{0} c}
\end{gathered}
$$


respectively. We note that the $\mathrm{z}$-components of the magnetic moment of the gamma photons do not depend on the angular frequency, $\omega$. Therefore we expect that the present result must be valid not only for gamma photons but also for any photon as well.

\section{Appendix III: Calculation of the Intrinsic Quantum Fluxes of Electron and Positron by Dirac Equation}

Solution of Dirac Equation for a free electron moving in a homegeneous magnetic field was given by Saglam et al. [6]:

$$
\begin{aligned}
& \left|e^{-\uparrow}\right\rangle=N f(t, \varphi, z)\left(\begin{array}{c}
F(-n, m+1, t) \\
0 \\
\frac{p_{z}}{E_{n}+M} F(-n, m+1, t) \\
\frac{2 n i \sqrt{\kappa} \eta \mathrm{e}^{i \varphi}}{\left(E_{n}+M\right)(m+1)} F(-n+1, m+2, t)
\end{array}\right) \\
& \left|e^{-} \downarrow\right\rangle=N f(t, \varphi, z)\left(\begin{array}{c}
0 \\
F(-n, m+1, t) \\
\frac{-2 m i \sqrt{\kappa} \mathrm{e}^{-i \varphi}}{\left(E_{n}+M\right) \eta} F(-n-1, m, t) \\
\frac{-p_{z}}{E_{n}+M} F(-n, m+1, t)
\end{array}\right)
\end{aligned}
$$

where we used the SI units in which $c=1$ (i.e. $M$ stands for the rest mass energy of the electron $m_{0} c^{2}$ ) The normalization constant, $N$ is given by:

$$
N=\sqrt{\frac{\kappa}{2 \pi}\left(1+\frac{M}{E_{n}}\right) \frac{(n+m) !}{n !(m !)^{2}}}
$$

and the function $f(t, \varphi, z)$ is defined as follows:

$$
f(t, \varphi, z)=t^{\frac{|m|}{2}} \mathrm{e}^{-\frac{t}{2}} \mathrm{e}^{i m \varphi} \mathrm{e}^{i p_{z} z}
$$

The corresponding energy eigenvalues are:

$$
\begin{gathered}
E_{n}^{2}(\uparrow)=4 \kappa n+M^{2}+p_{z}^{2} \\
E_{n}^{2}(\downarrow)=4 \kappa(n+1)+M^{2}+p_{z}^{2}
\end{gathered}
$$

To calculate the intrinsic quantum flux of a relativistic free electron in a uniform magnetic field within the framework of Dirac theory we shall follow a similar way that we followed earlier for the solution of Schödinger equation for an electron in hydrogen atom [4]: Namely, we shall first calculate the quantum flux through the probability current (particle current) density associated with the wave function of a free Dirac electron in a uniform magnetic field, then connect it to the flux element $\mathrm{d} \Phi_{z}(\rho, \phi)$ by the self-inductance $L_{e}$. The probabilty of electric current density for the circular motion along the $\phi$ direction is given by: 


$$
J(\rho, \phi, z)=\frac{i \hbar e}{2 M}\left\{\Psi^{*} \frac{\partial \Psi}{\rho \partial \phi}-\frac{\partial \Psi^{*}}{\rho \partial \phi} \Psi\right\} .
$$

Assuming that electron is moving in $x y$ plane we then write the, surface current element coming from the circular ring of radius $\rho$ and the thickness $\mathrm{d} \rho$ :

$$
J(\rho, \phi) \mathrm{d} \rho=\frac{i \hbar e}{2 M}\left\{\Psi^{*} \frac{\partial \Psi}{\partial \phi}-\frac{\partial \Psi^{*}}{\partial \phi} \Psi\right\} \frac{\mathrm{d} \rho}{\rho}
$$

Our next objective is to establish a quantized magnetic flux coming from the above current. A current element circulating around $\mathrm{z}$-axis in a circle of radius $\rho$ should enclose an induced magnetic flux element $\mathrm{d} \Phi_{z}(\rho, \phi)$ which is proportional to the current given by Equation (AIII-8):

$$
\mathrm{d} \Phi_{z}(\rho, \phi)=L_{e}(\rho) j(\rho, \phi) \mathrm{d} \rho
$$

where $L_{e}(\rho)$ is the self-inductance [4] given by:

$$
L_{e}(\rho)=M\left(\frac{2 \pi \rho}{e}\right)^{2}
$$

Substitution of Equations (AIII-8) and (AIII-10) into Equation (AIII-9) gives the total induced quantized magnetic flux:

$$
\Phi_{\mathrm{z}}(\text { ind })=\frac{2 \pi^{2} i \hbar}{e} \int\left\{\Psi^{*} \frac{\partial \Psi}{\partial \phi}-\frac{\partial \Psi^{*}}{\partial \phi} \Psi\right\} \rho \mathrm{d} \rho
$$

To calculate the total induced quantized magnetic fluxex for spin-up and spin-down electrons, we substitute the wave functions of $\left|e^{-} \uparrow\right\rangle$ and $\left|e^{-} \downarrow\right\rangle$ states separately from Equation (AIII-1) and Equation (AIII-2) in Equation (AIII-11):

$$
\begin{aligned}
& \Phi_{\text {ind }}(\uparrow)=\frac{-2 \pi \hbar}{e}(1+t)\left[\left(\frac{1}{1+t}\right) m+\frac{1}{2}\left(\frac{1-t}{1+t}\right)\right] \\
& \Phi_{\text {ind }}(\downarrow)=\frac{-2 \pi \hbar}{e}(1+t)\left[\left(\frac{1}{1+t}\right) m-\frac{1}{2}\left(\frac{1-t}{1+t}\right)\right]
\end{aligned}
$$

here we denote $t=\frac{M}{E_{n}}$. In the relativistic limit since $t \rightarrow 0$, then the spin dependent induced fluxes take the forms:

$$
\begin{aligned}
& \Phi_{\text {ind }}(\uparrow)=\frac{-2 \pi \hbar}{e}\left(m+\frac{1}{2}\right)=-\left(m+\frac{1}{2}\right) \frac{h}{e}=-\left(m+\frac{1}{2}\right) \Phi_{0} \\
& \Phi_{\text {ind }}(\downarrow)=\frac{-2 \pi \hbar}{e}\left(m-\frac{1}{2}\right)=-\left(m-\frac{1}{2}\right) \frac{h}{e}=-\left(m-\frac{1}{2}\right) \Phi_{0}
\end{aligned}
$$

In Equations (AIII-14a) and (AIII-14b) the spin dependent intrinsic fluxes correspond to $m=0$. Which are $\pm \frac{h}{2 e}= \pm \frac{\Phi_{0}}{2}$. So far we have used the SI units where $c=1$. For cgs units the flux quantum is $\Phi_{0}=\frac{h c}{e}=4.14 \mp 10^{-7} \mathrm{G} \cdot \mathrm{cm}^{2}$. So in cgs units the intrinsic fluxes that electron 
and positron possess are:

$$
\begin{gathered}
\Phi\left(e^{-} \downarrow\right)=\frac{h c}{2 e}=\frac{\Phi_{0}}{2} \\
\Phi\left(e^{-\uparrow}\right)=-\frac{h c}{2 e}=-\frac{\Phi_{0}}{2} \\
\Phi\left(e^{+} \downarrow\right)=-\frac{h c}{2 e}=-\frac{\Phi_{0}}{2} \\
\Phi\left(e^{+\uparrow}\right)=\frac{h c}{2 e}=\frac{\Phi_{0}}{2} .
\end{gathered}
$$

